of the tumour, and the current passed through it at intervals for about ten minutes. This procedure was repeated several times at subsequent sittings, so as to diminish the nutrition of the growth and gradually induce atrophy. In the intervals antiseptic and astringent douches were used. The patient returned to the country, and came up again in about a month, when his condition was satisfactory, and he had begun to breathe through the right nostril. On examination, a marked diminution was observed in the size of the tumour, in which the traces of the transfixions could still be seen. Encouraged by this result, the same treatment was continued at intervals during a month, the last transfixion being made in May, 1901. The patient was seen again three months later, when the tumour was found to be only as big as a hazel-nut and of a grayish-white colour. In the meantime the subjective noises had diminished. The patient was seen again in October, 1902, and as there still remained a small residuum of the tumour a last galvano-cautery transfixion was made. At the last examination of this patient in February, 1903, the nares and naso-pharynx were found perfectly free, and the subjective noises in the right ear had completely disappeared.

I have thought it useful to describe the results of this treatment as tending to confirm what has been already stated by Boulai-that the galvano-cautery perseveringly used can bring about the disappearance of a naso-pharyngeal fibroma. It is true that the slowness of this method does not allow it to be considered preferable, but, on the other hand, it has the great advantage of not exposing the patient to an operation of some gravity, such as the removal of the tumour through an opening made in the palate.

\title{
THE DEVELOPMENT AND MORPHOLOGY OF THE EAR.
}

\author{
By A. M. Paterson, M.D., \\ Professor of Anatomy, University College, Liverpoil.
}

WhiLe there is no organ in the body more complex in its development and structure, there is none which illustrates more forcibly the process of evolution from simpler to more complicated forms.

Three natural groups may be made of the cranial nerves: (1) Notor and myotomic - the third, fourth, sixth, and twelfth: (2) branchial-the fifth, seventh, ninth, tenth, and eleventh; and

* Communicated to the Otological Society of the United Kingdom. May 2 . 1903. 
(3) those associated with the sense organs of smell, sight, and hearing.

Without going into any comparison of the nerves in question, it is noteworthy that all the sense organs with which they are connected have certain fundamental characters in common. The nasal, optic, and auditory organs arise, to begin with, as epiblastic involutions from the surface of the head; each becomes secondarily connected with the buccal cavity by means of a cleft, pre-oral or branchial, and each gets an investment of mesoblast, forming a capsule, cartilaginous or fibrous.

Beyond these features in common, each organ proceeds in its own way to attain its mature condition.

The auditory organ arises as an invagination of the surface epiblast in series with the nasal pit and the lens; it is situated behind the position of the mouth, and dorsal to the first postoral visceral cleft.

In elasmobranchii the vesicle is permanently in relation with the surface by means of a ductus endolymphaticus, which is in direct communication with sea-water. In other vertebrates the vesicle becomes quite separated, and the duct ends blind, as the saccus endolymphaticus.

This epiblastic vesicle becomes immediately connected with the ganglionic outgrowth from the membrane which produces the auditory nerve, with its vestibular and cochlear subdivisions, and it is further differentiated into the parts which constitute the membranous labyrinth-saccule and cochlea, utricle and semicircular canals, the saccule and utricle being connected together by the ductus endolymphaticus. There are three semicircular canals in all vertebrates except Cyclostomi. In myxine the labyrinth is simple and tubular. In the lamprey only two canals occur. The cochlea is rudimentary in fishes; more elongated but simple in character in reptiles and birds ; it is only in mammals that it is spirally wound round the cochlear nerve, and among mammals its extent varies. In the rabbit, as in man, the cochlea has two and a half turns, in the ox three and a half turns, and in the pig four tirns, round the cochlear nerve.

The next occurrence in the formation of the organ is the enclosure of the auditory vesicle and its nerve in a mesoblastic capsule: in the case of the nasal and auditory organs, a capsule which is incorporated with the skeleton of the head and is developed in cartilage; in the case of the eye, a tibro-vascular capsule which is unattached to the cranial skeleton.

The otic capsule occurs throughont vertebrates. It acquires 
important connections with contiguous portions of the cranial skeleton, and in man produces the petro-mastoid bone. It assists in forming the base of the skull by its inclusion between the exoccipital and alisphenoid outgrowths of the para-chordal and trabecular cartilages. It is overlapped and eventually fused with the membranous vault of the skull, ont of which is produced the squamo-zygomatic bone, and the union of these two elements produces the mastoid antrum.

In relation to the contained auditory vesicle, the capsule in its growth shrinks from the membranous labyrinth, so as to lead to the formation of the perilymphatic space, which is in communication with the pericranial lymphatics by means of the aqueduct of the cochlea.

In fishes the above process represents the permanent stage of development of the ear, and sound is conveyed directly from and through the otic capsule to the auditory labyrinth.

The next step in the development of the organ occurs by the formation of a fenestra ovalis and a stapes, which may be associated with a columella reaching to the surface of the head and connected with a membrana tympani. In amphibia the stapes first appears. In the lemurs the base of the stapes is found; in cryptobranchus the columella occurs; and the fully-formed stapes and columella are present in reptiles and birds.

The tympanic cavity and Eustachian tube are essentially associated with the appearance of the ossicles. The stapes is developed as a special ossification round the stapedial artery, and is not related to the gill arches; it is formed in the mesoblastic tissue intervening between the otic capsule and the surface of the head.

The tympanic cavity is produced by the absorption of the mesoblastic tissue between the capsule and the surface, leading, pari passu, to the formation of the Eustachian tube and membrana tympani.

This is the second stage in the development and evolution of the ear, as represented in reptiles and birds.

The mammalian ear has already been noted as differentiated by the possession of a spiral cochlea. It is also characterized by important differences in the more external parts of the organ.

The tympanic ossicles are three in number. Added to the stapes are malleus and incus, the derivatives respectively of the articular and quadrate elements of the cartilage (Meckel's cartilage) of the mandibular arch. This is the first indication of the share of the branchial apparatus in the construction of the ear, and it is 
somewhat remarkable, as in mammals the branchial arches and clefts are subordinate and in a sense rudimentary. None of the clefts are pervious, and the first cleft and the first two branchial arches, mandibular and hyoid, are secondarily connected with the formation of the ear.

The cartilage of the first arch is essentially related to the formation of the lower jaw, and its proximal end, embedded close to the base of the skull between the otic capsule and the surface, gives rise to the malleus and incus, which are at first surrounded by embryonic mesoblastic tissue. As this becomes absorbed and swept away, the tympanic carity is produced, as already indicated.

The first branchial cleft, impervious in mammals, produces at its dorsal end the membrana tympani, lined externally by epiblast, and internally, after the formation of the tympanic cavity, by hypoblast.

The mammalian ear is further characterized by the possession of an external auditory meatus and a pinna, the former produced out of the superadded tympanic ring and by the growth of the deeper part of the pinna itself, the latter formed through the agency of the first and second branchial arches by the growth of confluent knobs or elevations surrounding the tympanic membrane.

The process of development thus very forcibly illustrates the line of evolution of the ear. There is first an invagination of the surface epiblast, connected with the brain and communicating with the surface (elasmobranchs); then a complete severance of connection with the surface (bony fishes); then the formation of tympanic cavity, membrana tympani, stapes, and columella in addition (reptiles and birds); then the addition of branchial elements, malleus and incus, external meatus, and pinna (mam. mals) ; and its complexity (and interest) are still further increased by the occurrence of mastoid antrum, mastoid process, and cells.

There are many obscure and interesting questions associated with the morphology of the ear. The spiral cochlea of mammals is a puzzle, although the curling of the tube may be only a mechanical arrangement for saving room. Another point that is obvious in the study of the subject is the quite secondary share taken in the formation of the ear by the branchial apparatus. Only in mammals are the branchial arches and first cleft concerned in its formation; and in mammals the association, although essentially important in the construction of the organs, is secondary both in time and order of evolution to the formation of the labyrinth and otic capsule.

$$
30-2
$$

